\title{
New objects do not capture attention without a sensory transient
}

\author{
Andrew Hollingworth \\ University of Iowa, Iowa City, Iowa \\ DANiel J. Simons \\ University of Illinois at Urbana-Champaign, Urbana, Illinois \\ AND \\ Steven L. Franconeri \\ Northwestern University, Evanston, Illinois
}

\begin{abstract}
Attention capture occurs when a stimulus event involuntarily recruits attention. The abrupt appearance of a new object is perhaps the most well-studied attention-capturing event, yet there is debate over the root cause of this capture. Does a new object capture attention because it involves the creation of a new object representation or because its appearance creates a characteristic luminance transient? The present study sought to resolve this question by introducing a new object into a search display, either with or without a unique luminance transient. Contrary to the results of a recent study (Davoli, Suszko, \& Abrams, 2007), when the new object's transient was masked by a brief interstimulus interval introduced between the placeholder and search arrays, a new object did not capture attention. Moreover, when a new object's transient was masked, participants could not locate a new object efficiently even when that was their explicit goal. Together, these data suggest that luminance transient signals are necessary for attention capture by new objects.
\end{abstract}

Where people attend determines, to a large extent, what they perceive, remember, and act upon (for reviews, see Luck \& Vecera, 2002; Pashler, 1998). Understanding human behavior therefore requires understanding the factors that control where attention is directed in a scene. In most cases, top-down control is exerted over the allocation of attention, so that objects relevant to the current task can be selected (Hayhoe, 2000; Hollingworth, 2009; Land, Mennie, \& Rusted, 1999; Torralba, Oliva, Castelhano, \& Henderson, 2006). However, salient stimuli can recruit attention independently of, or even in opposition to, an observer's goals (e.g., Franconeri, Simons, \& Junge, 2004; Yantis \& Jonides, 1984, 1990). Such attention capture plays an important role in ensuring that unexpected and behaviorally relevant visual events are processed efficiently.

Two competing hypotheses have been proposed to explain how attention is captured by salient visual events. Under the transient hypothesis (e.g., Franconeri, Hollingworth, \& Simons, 2005; Yantis \& Jonides, 1984), attention is drawn by the abrupt sensory transients created when an object undergoes a salient change. For example, if an object moves into view, the motion transient generated by that object will capture attention (Abrams \& Christ, 2005; Franconeri \& Simons, 2003). Under the new-object hypothesis (Yantis, 2000; Yantis \& Hillstrom, 1994), the only event that is proposed to capture attention reliably is the appearance of a new object in the visual field. Critically, capture under this view does not depend on the sensory transient created by the object's appearance.

In addition to these two alternatives, certain salient static features, such as a unique color among homogeneous distractors (Theeuwes, 1992, 2004) or an object that matches the current content of visual working memory (Hollingworth \& Luck, 2009; Olivers, Meijer, \& Theeuwes, 2006; Soto, Heinke, Humphreys, \& Blanco, 2005), can recruit attention in a seemingly automatic fashion, but whether or not these examples represent truly automatic, stimulus-driven attention capture remains a topic of considerable debate (Downing \& Dodds, 2004; Folk, Remington, \& Johnston, 1992; Leber \& Egeth, 2006; Woodman \& Luck, 2007).

Current evidence generally favors the hypothesis that transient events play a central role in attention capture and that new objects do not reliably capture attention without a sensory transient (Franconeri et al., 2005). Salient changes to previously visible (i.e., old) objects, including object motion, looming, luminance change, and contrast polarity change (Abrams \& Christ, 2005; Enns, Austen, Di Lollo, Rauschenberger, \& Yantis, 2001; Franconeri \& Simons, 2003; Thomas \& Luck, 2000), reliably capture attention. Thus, attention capture is not limited to the appearance of a new object. In addition, a new object does not appear to be sufficient for attention capture when it does not create a

A. Hollingworth, andrew-hollingworth@uiowa.edu 
unique sensory transient (Brockmole \& Henderson, 2005a, 2005b; Chua, 2009; Franconeri et al., 2005).

Franconeri et al. (2005) modified the classic irrelevant singleton task (Yantis \& Jonides, 1984) to eliminate the transient associated with the addition of a new object. In the standard version of this task, participants see a set of figure eight placeholders. Segments of the placeholders are deleted to form letters, and, simultaneously, a new letter is added to the display at a location that previously had no placeholder. When the new letter is the search target, response times (RTs) are rapid and show minimal effects of search set size, suggesting that attention was directed first to the new object (i.e., it was detected efficiently and captured attention). When the target is one of the old objects, RTs increase with increasing set size, because participants must search serially through the array to find the target.

In the modified version of this task (Franconeri et al., 2005), an annulus (ring) surrounded the initial placeholder array. The annulus contracted so that it briefly occluded the placeholders and then contracted further to reveal the search array. The new object was added during the instant of maximum occlusion. Thus, a new object was added, but it did not create a unique onset transient, because its addition occurred during the brief occlusion event. In a control condition, the annulus passed behind the array so that the transient created by the new object was visible. Attention capture by a new object was observed only in the control condition, in which the onset transient was visible. No capture occurred when the new object's transient was obscured by the occluder. Similarly, when a new object was added to a photograph of a natural scene during an eye movement (Brockmole \& Henderson, 2005b), thereby masking the transient caused by its appearance, the eyes were not drawn reflexively to the new object. Both results suggest that new objects capture attention only when they create a unique sensory transient.

In contrast, a recent study by Davoli, Suszko, and Abrams (2007) presented evidence for attention capture by a new object in the absence of a unique transient signal. In this study, placeholders were displayed, followed by an interstimulus interval (ISI) of 100 or $400 \mathrm{msec}$ and then by the search display. The ISI ensured that the new object did not create a unique transient, because after the ISI, all search letters generated an onset transient. Despite separation of placeholder and search arrays by an ISI, the slope relating RT to set size was lower when the target was a new object than when the target was an old object, suggesting that the new object captured attention in the absence of a unique sensory transient.

These results pose a strong challenge to the transient hypothesis, but they are somewhat puzzling, given what is known about visual memory and change detection. In order for a new object to capture attention across an ISI, observers would need to be able to detect the change in the array (i.e., to detect the new object that was added to the array). Successful change detection would require participants to encode the locations of the preview objects into visual working memory (VWM), retain them across the ISI, and compare them with objects in the search array. ${ }^{1}$
The one object in the search array that did not correspond to a remembered location could then be detected as the new object, and it might recruit attention (Cole, Kentridge, \& Heywood, 2004). But this type of process would be limited by the capacity of VWM (Luck \& Vogel, 1997; Phillips, 1974). If the preview display contained more objects than could be maintained in VWM, the new object could not be detected reliably. Thus, the ability to detect the presence of a new object across an ISI-ignoring for now the question of whether such detection generates attention capture - should be limited to cases in which only a small number of objects are visible. In complex, real-world environments containing scores of individual objects, the introduction of a new object across a brief disruption would rarely be detected. Such insensitivity to change has been demonstrated consistently in the literature on change blindness (for reviews, see Hollingworth, 2008; Simons \& Rensink, 2005). If people cannot reliably detect the addition of a new object in a scene across a blank ISI or other perceptual disruption when change detection is their explicit task, it is unlikely that the addition of a new object across a blank ISI would reliably capture attention when it is irrelevant to the task. ${ }^{2}$

In addition, interpretation of the Davoli et al. (2007) results is limited by the fact that the experiments used atypical instructions. Participants were told that new objects would appear, but they were never instructed that new objects were no more likely to be the target than were old objects (C. C. Davoli, personal communication, May 7, 2008). The latter instruction is standard in the literature, because informing participants that new objects are task irrelevant is necessary to ensure that they do not strategically monitor for new objects. Without it, no firm inferences can be drawn regarding involuntary attention capture, because the participants may have treated new objects as task relevant.

To establish whether a new object captures attention without a sensory transient, we conducted four experiments probing participants' sensitivity to the addition of a new object to a search array across an ISI. In Experiment 1, we sought to replicate the Davoli et al. (2007) finding of capture across an ISI. In three subexperiments, we were unable to replicate their results. In Experiments 2 and 3, we modified the capture paradigm to provide an even more liberal test of new-object detection and potential capture across an ISI. In addition, we compared new-object capture across an ISI (when the new object does not create a unique sensory transient) with the standard capture paradigm that has no ISI (when the new object does create a unique sensory transient). We found capture only when the new object created a unique transient. In Experiment 4, we tested whether the addition of a new object can even be detected efficiently across an ISI by making the new object the target of search on every trial. Consistent with the literatures on VWM and change blindness, we found that participants could not reliably locate the new object in the search display, making it unlikely that such an event could automatically capture attention. Together, these results support the hypothesis that sensory transients play a central role in attention capture and that new objects do not reliably capture attention without a unique sensory transient (Franconeri et al., 2005). 


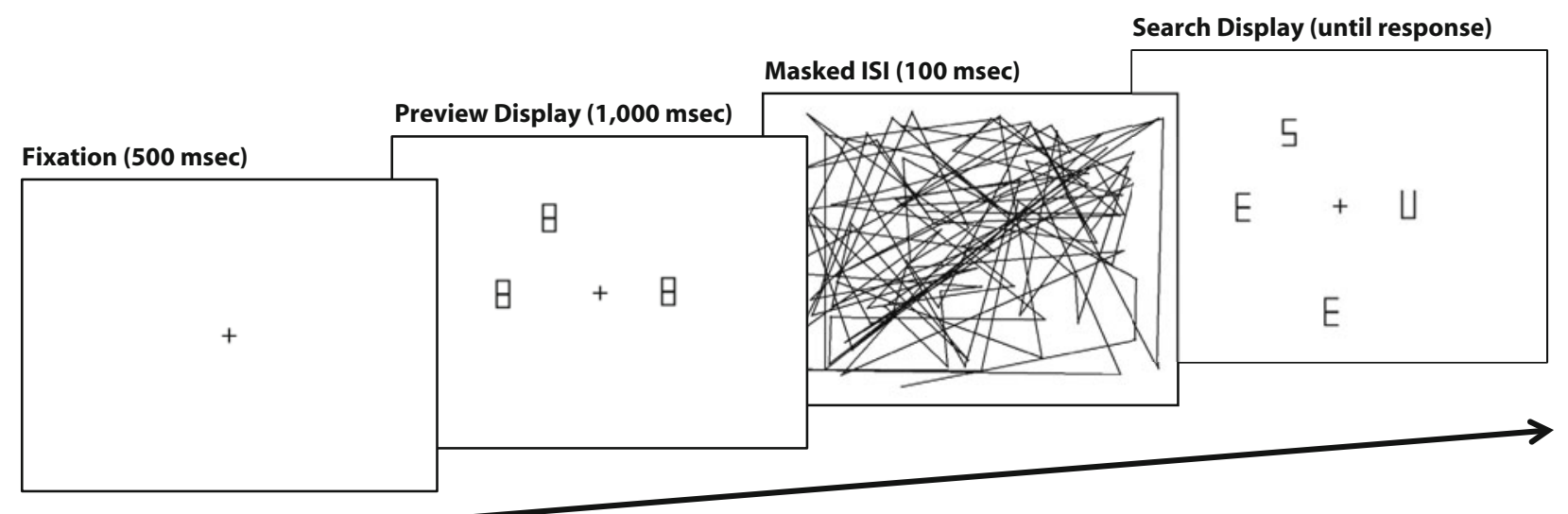

Figure 1. Sequence of events in a trial in Experiment 1, replicating the method of Davoli, Suszko, and Abrams (2007). Upon the appearance of the search display, participants searched for a target letter ("H" or "S"). A new object was added to each search array. In the example trial illustrated here, the target ("S") is one of the old objects. ISI, interstimulus interval.

\section{EXPERIMENT 1}

In Experiment 1, we sought to replicate the Davoli et al. (2007) experiments by matching, as closely as possible, their stimuli and procedure (see Figure 1). In this variant of the classic irrelevant feature paradigm (Yantis \& Jonides, 1984), participants first saw a placeholder array of either three or seven figure eight placeholders. The placeholder array was followed by a 100 -msec masked ISI and a search array. In the search array, placeholders were replaced by letters, and one new object was added to the display. Search set size was therefore either four or eight letters. One target letter was present, an "H" or an "S," and the participants responded to indicate target identity. The distractors were either an "E" or a "U," randomly chosen. The new object in the search array was no more likely to be the target than were any of the old objects.

In three subexperiments, we manipulated instructions to determine whether the atypical instructions in Davoli et al. (2007) contributed to their results. In Experiment 1A, we replicated the Davoli et al. instructions, informing the participants about the appearance of new objects, but not informing them that this attribute was task irrelevant. In Experiment 1B, we used the standard instructions in the attention capture literature: The participants were told that the new object was no more likely to be the target than were any of the other letters in the array. In Experiment $1 \mathrm{C}$, we sought to provide a particularly strong test of the potential effect of instructions by informing the participants that there would be new objects and telling them that they should pay "particular attention" to new objects. These instructions encouraged the participants to strategically monitor for new objects.

\section{Method $^{3}$}

Participants. The participants in all the experiments were between 18 and 30 years of age, were recruited from the University of Iowa community, and were naive with respect to the hypotheses under investigation. Each participant completed only one experi- ment. They received either course credit or pay. All the participants reported normal or corrected-to-normal vision. Twenty different participants completed each of the three subexperiments.

Stimuli and Apparatus. The background was white, and the placeholders, letters, and central fixation cross were black. The placeholders in the preview display were block figure eights $\left(0.8^{\circ}\right.$ wide $\times 1.6^{\circ}$ high) . There were either three placeholders (search set size 4 condition) or seven placeholders (search set size 8 condition). Placeholders were randomly positioned within a $21.6^{\circ} \times 15.2^{\circ}$ region at screen center, with the constraints that the edges of two placeholders could not be closer than $0.4^{\circ}$ and that no placeholders could appear within a $0.8^{\circ} \times 0.8^{\circ}$ region around the central fixation cross. In the search array, the placeholders were replaced by letters. Old letters were presented in the same locations as those occupied by the placeholders in the preview display and were formed by deleting segments of the placeholders. In addition, a new letter was added to the display at a randomly chosen location. The target letter was either an " $\mathrm{H}$ " or an "S," with distractors randomly chosen from "E" and "U." The mask appearing between the preview and search displays was constructed, before the beginning of each trial, by connecting a series of 100 randomly chosen points within the search region with black lines. During the trial, the mask was presented as a single image.

The stimuli were displayed on a 17 -in. CRT monitor at a viewing distance of $80 \mathrm{~cm}$, maintained by a forehead rest. Responses were collected using a serial button box. The experiment was controlled by E-Prime software (Schneider, Eschmann, \& Zuccolotto, 2002). To ensure precise stimulus timing, all the images appearing on a trial were loaded into graphics memory before the trial began. Stimulus presentation was synchronized with the monitor's vertical retrace. The refresh rate was set at $100 \mathrm{~Hz}$.

Procedure. The participants pressed a pacing button to initiate each trial. A central fixation cross appeared for $500 \mathrm{msec}$, followed by the preview display for $1,000 \mathrm{msec}$, then by the mask (ISI) for $100 \mathrm{msec}$, and finally by the search display. The participants pressed one of two buttons to indicate the presence of "H" or "S." They were instructed to respond as quickly as possible without making a significant number of errors. There was a 1,000-msec delay between trials. During this interval, the participants received feedback on incorrect trials, with a central "Incorrect" message presented on the screen in red. In addition, if the participants responded too quickly $(<100 \mathrm{msec})$ or too slowly $(>1,500 \mathrm{msec})$, the message "Too fast!" or "Too slow!" was presented.

Each subexperiment was a 2 (set size) $\times 2$ (target type: new, old) $\times 2$ (target: "H" or "S") design. The participants first com- 
Table 1

Mean Accuracy (in Percentages) in Experiment 1

\begin{tabular}{ccc}
\hline & \multicolumn{2}{c}{ Set Size } \\
\cline { 2 - 3 } Condition & 4 & 8 \\
\hline Experiment 1A & & \\
Target old & 96.5 & 95.5 \\
Target new & 97.5 & 95.0 \\
Experiment 1B & & \\
Target old & 96.8 & 96.1 \\
Target new & 95.4 & 95.7 \\
Experiment 1C & & \\
Target old & 96.8 & 96.3 \\
Target new & 97.0 & 97.5 \\
\hline
\end{tabular}

pleted 10 practice trials, followed by seven blocks of experiment trials. Each block consisted of 64 trials: 32 at set size 4 and 32 at set size 8 . For set size 4 , the new object was the target on 8 trials $(25 \%)$. For set size 8 , the new object was the target on 4 trials $(12.5 \%)$. Within-block trial order was determined randomly. The participants completed a total of 448 experiment trials.

In Experiment 1A, the participants were given instructions that matched the basic features of the instructions used by Davoli et al. (2007; C. C. Davoli, personal communication, May 7, 2008). They were told that "there will always be one 'new letter' that appears in a location that had not contained a figure 8," but they were not told that the new letter was no more likely to be the target than were the other letters.

In Experiment 1B, the participants were given standard instructions for an attention capture experiment. As in Experiment 1A, they were informed about the addition of a new letter, but they were also told that "this letter is no more likely to be the target than any of the other letters."

In Experiment $1 \mathrm{C}$, the instructions were the same as those in Experiment 1A, except that the participants were also told to "pay particular attention to the new letter."

\section{Results}

There was no main effect of target type (new, old) on accuracy in any of the three subexperiments, nor was there an interaction between target type and set size (see Table 1). In Experiment 1A, there was a reliable effect of set size on accuracy, with higher accuracy at set size 4 than at set size $8[F(1,19)=8.67, p=.008]$, although the numerical difference was small $(<2 \%)$.

In all the experiments, RTs greater than $2.5 \mathrm{SDs}$ from each participant's mean in each condition were eliminated as outliers. No more than $3.2 \%$ of the data was eliminated in any experiment.

The RT data are shown in Figure 2. The standard pattern indicating attention capture is a shallower search slope in the target-new condition than in the target-old condition (i.e., an interaction between target type and set size). In addition, lower overall RTs in the target-new condition typically result in a main effect of target type. This full pattern was observed in none of the three subexperiments, although there was a slope difference in Experiment 1C, in which the participants were instructed to "pay particular attention" to new objects (see Figure 2).

Experiment 1A: Davoli et al. (2007) instructions. No attention capture was observed. Search rate was not reliably different in the target-new $(22.7 \mathrm{msec} /$ item $)$ and target-old $(21.5 \mathrm{msec} /$ item $)$ conditions $(F<1)$. Collapsing across set size, responses were no faster in the target-new

\section{Experiment 1A}

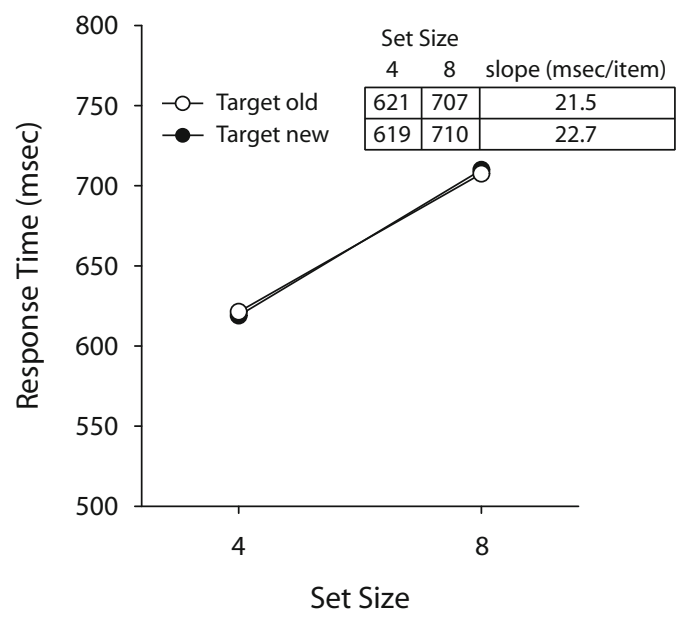

Experiment 1B

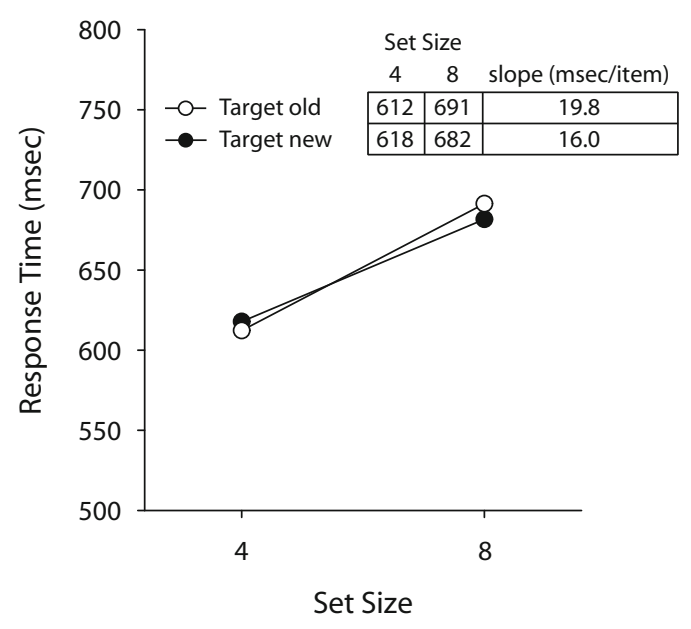

Experiment 1C

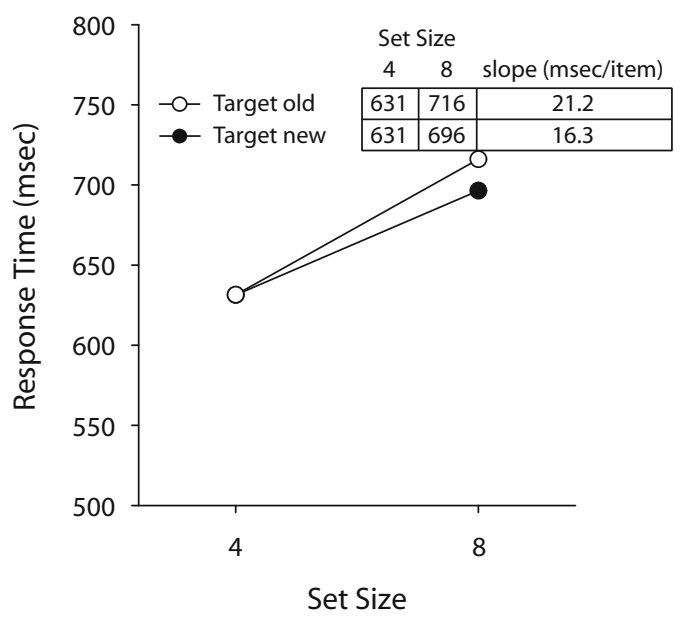

Figure 2. Experiment 1: Mean response time data as a function of target type (target old, target new) and set size in each of the three Experiment 1 subexperiments. The inset tables report the specific data points plotted in the graphs and the search slopes for the target-old and target-new conditions. 
condition than in the target-old condition $(F<1)$. This experiment had sufficient power to detect a slope difference of $7.9 \mathrm{msec} /$ item, which is considerably smaller than the slope difference typically found in cases of attention capture (Franconeri et al., 2005; Yantis \& Jonides, 1984).

Experiment 1B: Standard instructions. Again, no attention capture was observed. Search rate was not reliably different in the target-new $(16.0 \mathrm{msec} /$ item $)$ and target-old $(19.8 \mathrm{msec} /$ item $)$ conditions $[F(1,19)=2.45$, $p=.13]$. Collapsing across set size, responses were no faster in the target-new condition than in the target-old condition $(F<1)$. There was sufficient power to detect a slope difference of $5.1 \mathrm{msec} /$ item.

Experiment 1C: Instruction to attend selectively to new objects. Search was reliably more efficient in the target-new condition $(16.3 \mathrm{msec} /$ item $)$ than in the targetold condition (21.2 msec/item) $[F(1,19)=5.09, p=.04]$. Collapsing across set size, there was a trend toward faster responses in the target-new condition than in the targetold condition $[F(1,19)=3 \cdot 27 . p=.09]$. In absolute terms, though, the slope difference was small and fell short of the slope differences typically observed in unambiguous cases of attention capture.

\section{Discussion}

In three subexperiments, we were unable to replicate the full pattern of results observed by Davoli et al. (2007). The absence of slope difference between the target-new and target-old conditions when standard instructions were used (Experiment 1B) suggests that a new object across an ISI does not capture attention involuntarily. The absence of a slope difference when the Davoli et al. instructions were used (Experiment 1A) suggests that the specific instructions used by Davoli et al. might not have played a significant role in generating their original effect. However, the results of Experiment 1C, in which the participants were instructed to "pay particular attention" to new objects, demonstrate that, in general, instructions that encourage participants to attend to new objects can generate a pattern of data suggestive of attention capture. It is likely that, in Experiment 1C, the participants explicitly monitored for new objects (see also Chua, 2009, and the present Experiment 4). This effect of instruction highlights the need to ensure that in attention capture studies, participants do not construe any advantage to strategically prioritizing new objects.

\section{EXPERIMENT 2}

In Experiment 1, we did not replicate the results of Davoli et al. (2007) under experimental conditions directly modeled after their experiments. In Experiment 2, we sought to expand our test of the conditions under which attention capture might possibly be found across an ISI. The irrelevant singleton paradigm was modified so as to generate the most favorable conditions for observing new object capture across an ISI, if such capture exists. Set size was reduced so that it did not exceeded VWM capacity, and the duration of the mask appearing between the two arrays was reduced to $40 \mathrm{msec}$. We address each of these features in turn.

\section{Set Size and VWM Capacity}

In the larger set size of Experiment 1, the number of objects to maintain across the ISI (seven) was likely to have exceeded VWM capacity for locations (Jiang, Olson, \& Chun, 2000). If the number of objects to remember never exceeded VWM capacity, perhaps the change introduced by a new object would be detected efficiently and could then recruit attention automatically. This possibility is broadly consistent with a recent study (Hyun, Woodman, Vogel, Hollingworth, \& Luck, 2009) in which participants performed a color change detection task and the memory set size was manipulated (two, three, or four objects). After the ISI separating the memory and test arrays, attention was shifted to the changed item in a manner that was largely independent of the number of objects entering into the comparison. That is, the latency of the shift of attention to the changed object (as indexed by the N2PC ERP component) was no greater for set size 4 than for set size 2 . Hyun et al. argued that color changes can behave as an elementary feature in vision and pop out in visual search. Of course, this would apply only when the memory set size does not exceed the capacity of VWM. Furthermore, efficient detection of the color change does not necessarily mean that this type of change involuntarily captures attention (Yantis \& Egeth, 1999), because participants had every incentive to shift attention to the changed object.

However, the Hyun et al. (2009) results do raise the possibility that the change created by the addition of a new object across an ISI may be detected efficiently if the set size is within VWM capacity. Indeed, new objects can be prioritized for change detection across an ISI using relatively small set sizes (Cole, Kuhn, \& Liversedge, 2007). And, if the new object were detected efficiently, it might then capture attention. If observed, this would constitute a highly limited form of attention capture, however. In realworld scenes, the number of visible objects almost always exceeds the capacity of VWM (hence, the phenomenon of change blindness), and a new object will not be detected reliably across an ISI or other perceptual disruption (Brockmole \& Henderson, 2005b).

To test this possibility, in Experiment 2, we reduced the set size in the irrelevant singleton task to ensure that in all the conditions, the number of objects to remember across the ISI did not exceed VWM capacity (see also Franconeri et al., 2005). On each trial (see Figure 3), the participants saw three figure eight placeholders arrayed in a triangular configuration, followed by an ISI and then by a search array of two, three, or four letters. One of the letters appeared in a location where no placeholder had been (i.e., it was a new object). The new letter could appear in any of the three gaps between the placeholders, ensuring that the participants could not reliably predict the location of the new object. On $1 / n$ trials (where $n$ is search set size), the new object was the target. The participants were informed that the new object was no more likely to be the target than were any of the other array letters. Finally, we included a 0 -msec ISI control condition, in which the new object created a unique onset transient. This condition is equivalent to the classic irrelevant singleton paradigm (Yantis \& Jonides, 1984), enabling us to 


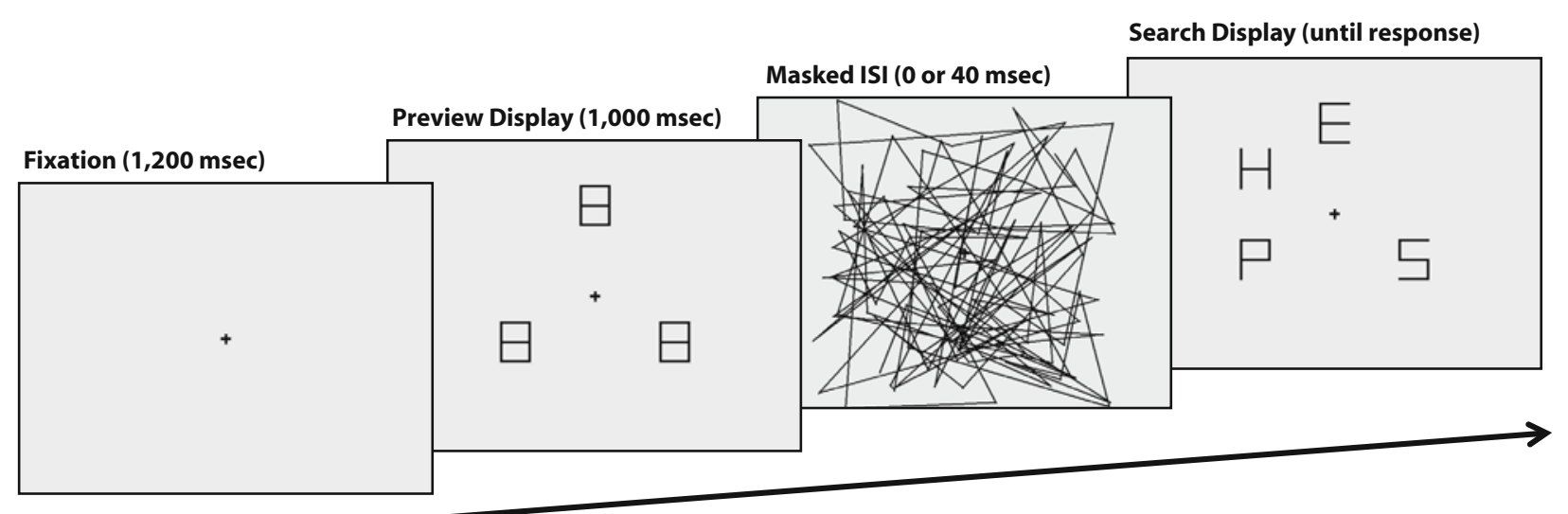

Figure 3. Sequence of events in a trial in Experiment 2. Upon the appearance of the search display, participants searched for a target letter ("H" or "U"). A new object was added to each search array. In the example trial illustrated here, the target ("H") is the new object. ISI, interstimulus interval.

compare the ISI results with results from a paradigm known to produce attention capture by a new object.

\section{ISI Duration}

The shortest ISI duration used by Davoli et al. (2007) was $100 \mathrm{msec}$, and this was the ISI duration used in Experiment 1. It is possible, however, that a 100 -msec gap is too long to establish the continuity between objects presented in the placeholder and search arrays and, thus, too long to reliably support new-object capture. Object persistence can be computed over ISIs as long as 2,700 msec (Mitroff \& Alvarez, 2007), which would suggest that a 100 -msec ISI should not place any significant constraints on the computation of object correspondence. However, there is evidence that object representations functional in attention capture might be less robust. Yantis and Gibson (1994) used the standard irrelevant feature task, with no ISI between the placeholder and search arrays. However, one placeholder was offset for a variable duration prior to the search array and then appeared again as a letter in the search array. The question of interest was whether the reappearing object would capture attention. When the single placeholder disappeared for more than $100 \mathrm{msec}$ prior to search, there was evidence of robust capture by the reappearing object, but no systematic evidence of capture for shorter gaps. Yantis and Gibson argued that a gap of $100 \mathrm{msec}$ or greater causes the reappearing object to be treated as a new object (rather than as a continuation of the placeholder), thus attracting attention. ${ }^{4}$ In Experiment 1, the fact that all objects offset for $100 \mathrm{msec}$ might have caused all objects to be treated as "new" across the ISI potentially explains the lack of capture. To address this issue, we reduced the ISI duration in Experiment 2 to $40 \mathrm{msec}$.

\section{Method}

Participants. Thirty-two participants completed the experiment.

Stimuli and Apparatus. The background was light gray, and the placeholders, letters, and fixation cross were black. The placeholders were block figure eights $\left(1.69^{\circ}\right.$ wide $\times 2.26^{\circ}$ high $)$. In the preview display, three placeholders were arranged at the vertices of an upward- or downward-pointing equilateral triangle, $5.02^{\circ}$ from a central fixation point. In the search display, old letters were presented at the same locations as the placeholders in the preview display. A new letter was present in each search display, and it appeared in between two placeholder positions. There was always one target, either an "H" or a "U," in the display, and distractors were randomly sampled without replacement from the remaining letters ("S," "P," and "E"). For searches with two or three letters, either two or one of the original placeholders was removed from the search display. The mask was created in the same manner as in Experiment 1, except that it was constrained to a $17^{\circ} \times 17^{\circ}$ central region of the screen, which easily covered the object stimuli.

Procedure. On each trial, a central fixation cross appeared for $1,200 \mathrm{msec}$, followed by the preview display for $1,000 \mathrm{msec}$ and the search display. There was either no ISI between the preview and search displays or a 40-msec masked ISI. The participants pressed one of two buttons to indicate the presence of "H" or "U." Incorrect responses resulted in an error message and a short delay. The participants were instructed to respond as quickly and as accurately as possible. In addition, they were informed that new objects were no more likely to be the target than were old objects.

The experiment was a 2 (ISI: 0 or $40 \mathrm{msec}) \times 3$ (set size) $\times$ 2 (target type: new, old) $\times 2$ (target: "U" or "H") design. After instructions, the participants completed 8 practice trials in each of the ISI conditions. The practice session was followed by four blocks of trials, two blocks of each ISI condition presented in interleaved order. The starting ISI condition was counterbalanced across participants. Within each block, the participants first completed 3 buffer trials (randomly selected from the current block), followed by 90 experiment trials: 20 trials of set size 2, 30 of set size 3 , and 40 of set size 4. At each set size, the new object was the target on 10 trials. Within-block trial order was determined randomly. The participants completed a total of 360 experiment trials.

\section{Results and Discussion}

Accuracy data are reported in Table 2. The mean error rate was $2.1 \%$. In the 40 -msec ISI condition, there was no effect of set size $[F(2,62)=1.50, p=.23]$. There was a marginal effect of target type $[F(1,31)=3.56, p=.07]$, with $98.2 \%$ correct when the target was new and $97.3 \%$ correct when the target was old. There was no interaction between set size and target type $(F<1)$. In the 0 -msec ISI condition, there was no effect of set size $[F(2,62)=1.23$, $p=.30]$. The participants were statistically more accurate when the target was a new object $(98.5 \%)$ than when it 
was an old object $(97.5 \%)[F(1,31)=9.62, p=.004]$, but the numerical difference was minor. Target type and set size did not interact $(F<1)$.

RT data are shown in Figure 4. Search slopes were estimated by linear regression. In the 40-msec ISI condition, when the new object did not create a unique transient, no attention capture was observed. Search rate was not reliably different in the target-new $(25.1 \mathrm{msec} /$ item $)$ and target-old $(23.9 \mathrm{msec} /$ item $)$ conditions $(F<1)$. Collapsing across set size, responses were no faster in the targetnew condition than in the target-old condition $(F<1)$.

In the 0 -msec ISI control condition, the new object created a unique onset transient, and the new object captured attention. Search rate was shallower in the target-new
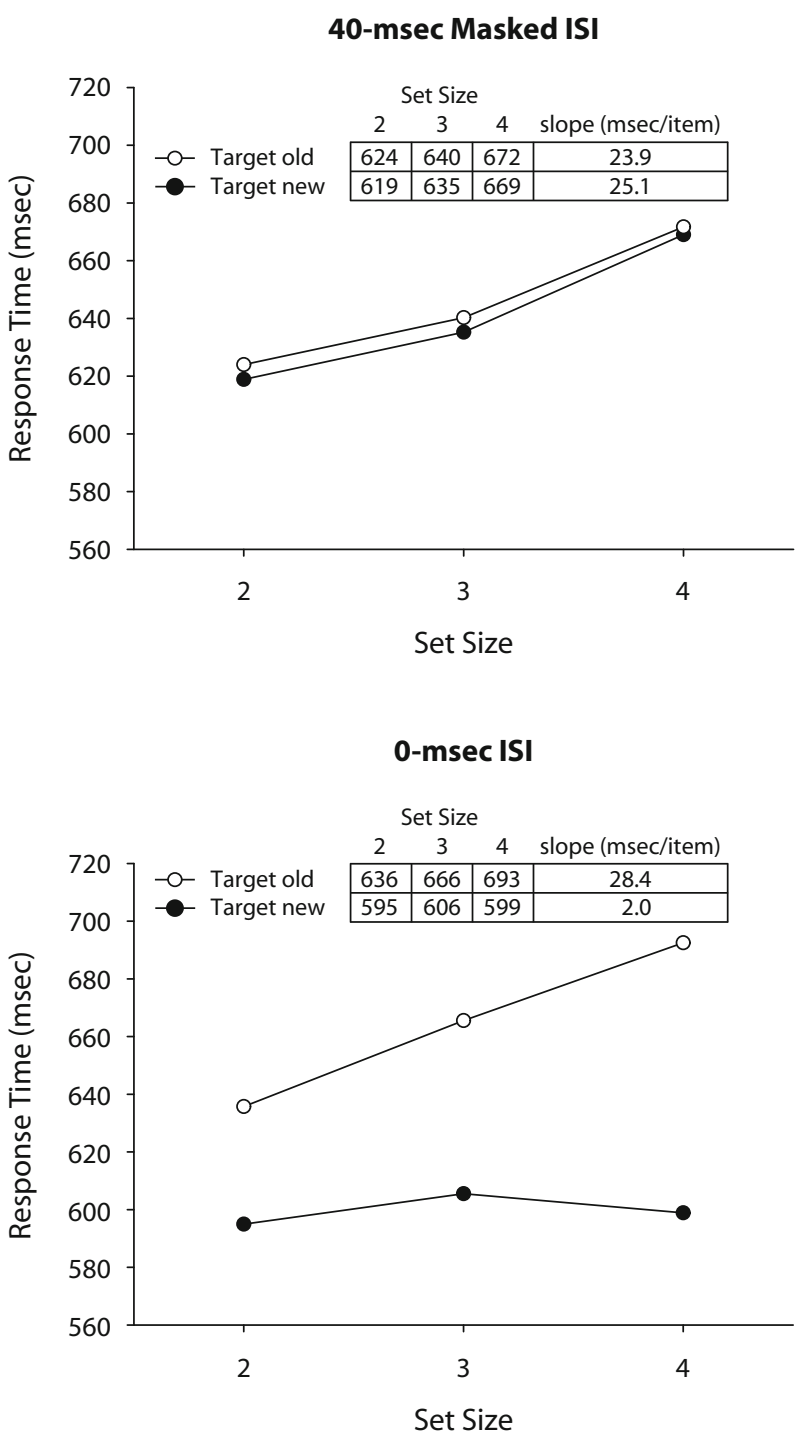

Figure 4. Experiment 2: Mean response time data as a function of target type (target old, target new) and set size. The top panel shows the data from the 40-msec interstimulus interval (ISI) condition, and the bottom panel the data from the 0-msec ISI control condition. The inset tables report the specific data points plotted in the graphs and the search slopes for the target-old and targetnew conditions.
Table 2

Mean Accuracy (in Percentages) in Experiment 2

\begin{tabular}{cccc}
\hline & \multicolumn{3}{c}{ Set Size } \\
\cline { 2 - 4 } Condition & 2 & 3 & 4 \\
\hline 40-msec Interstimulus Interval & & & \\
Target old & 96.6 & 97.9 & 97.4 \\
Target new & 98.0 & 98.4 & 98.1 \\
0-msec Interstimulus Interval & & & \\
$\quad$ Target old & 98.0 & 97.4 & 97.2 \\
Target new & 98.7 & 98.0 & 98.7 \\
\hline
\end{tabular}

condition $(2.0 \mathrm{msec} /$ item $)$ than in the target-old condition $(28.4 \mathrm{msec} /$ item $)[F(1,31)=28.0, p<.001]$. Collapsing across set size, responses were faster in the target-new condition than in the target-old condition $[F(1,31)=$ $179.3, p<.001]$.

Consistent with capture by the new object in the 0 -msec ISI condition, but not in the $40-\mathrm{msec}$ ISI condition, the slope data produced a reliable interaction between ISI condition and target type (old, new) $[F(1,31)=5.0, p=.03]$.

Despite the fact that the participants should have been able to maintain all three placeholders in VWM across the ISI, and despite the very short ISI duration, there was no evidence of capture by the new object in the 40-msec ISI condition. VWM capacity and ISI duration do not appear to play a central role in controlling attention capture in this type of paradigm. As long as the unique transient associated with the new object is eliminated, no capture is observed. In contrast, when the new object created a unique transient in the 0 -msec ISI control condition, it captured attention, consistent with other studies showing that the onset of an object reliably captures attention when that onset creates a unique transient signal (Franconeri et al., 2005; Theeuwes, 1991; Yantis \& Jonides, 1984, 1990). The latter effect demonstrates that the paradigm was well constructed to observe attention capture and that the absence of capture in the 40-msec ISI condition was not caused by insensitivity of the general method to effects of attention capture.

\section{EXPERIMENT 3}

In Experiments 1 and 2, the ISI was filled by a pattern mask, as in Davoli et al. (2007). It is possible that a pattern mask might actually have impaired the participants' ability to establish object continuity across an ISI-generating a salient sensory signal that interfered with the maintenance of object representations - and thus impaired their ability to establish which object was new and which objects were old in the search array. In Experiment 3, we eliminated the mask, using a blank (fixation cross only) ISI of $200 \mathrm{msec}$ (a value that lies between the two ISI durations used by Davoli et al., 2007: 100 and $400 \mathrm{msec}$ ). Otherwise, the method was identical to that in Experiment 2. As in Experiment 2, a 0 -msec ISI control condition was included, in which we expected to observe robust capture.

\section{Method}

Participants. Thirty-two participants completed the experiment. 
Stimuli, Apparatus, and Procedure. The experiment was identical to Experiment 2, except that the 40-msec masked ISI condition was replaced by a 200 -msec blank ISI condition. The blank ISI consisted of the background gray with a central fixation cross (to ensure that there was no offset other than the placeholders themselves).

\section{Results and Discussion}

Accuracy data are reported in Table 3. The mean error rate was $1.4 \%$. In the 200 -msec ISI condition, there was a marginal effect of set size $[F(2,62)=2.72, p=.07]$, with accuracy increasing with increasing set size. There was no effect of target type $[F(1,31)=0.17, p=.68]$ and no interaction between set size and target type $[F(2,62)=$ $0.30, p=.74]$. In the 0 -msec ISI condition, there was a
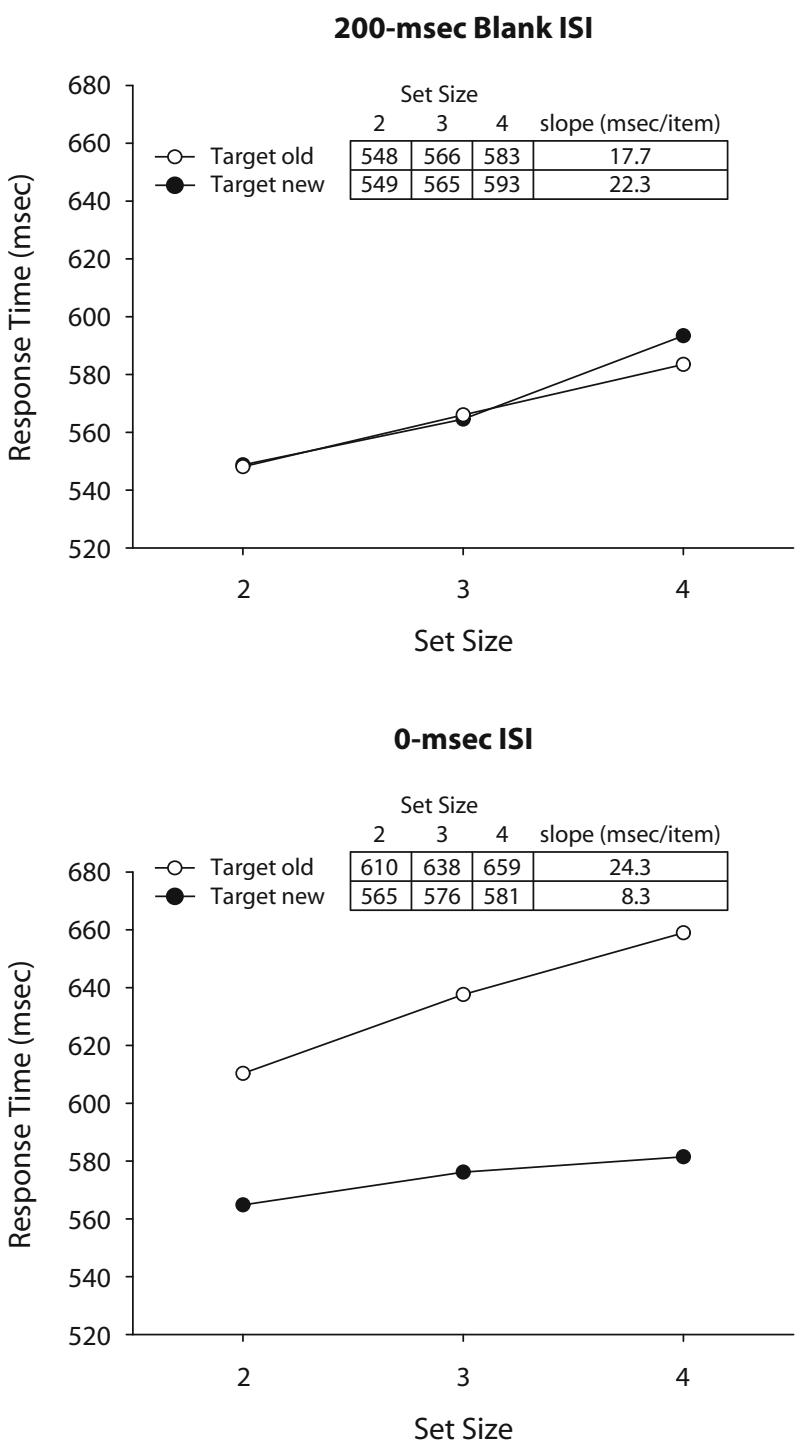

Figure 5. Experiment 3: Mean response time data as a function of target type (target old, target new) and set size. The top panel shows the data from the 200-msec interstimulus interval (ISI) condition, and the bottom panel the data from the 0-msec ISI control condition. The inset tables report the specific data points plotted in the graphs and the search slopes for the target-old and target-new conditions.
Table 3

Mean Accuracy (in Percentages) in Experiment 3

\begin{tabular}{lccc}
\hline & \multicolumn{3}{c}{ Set Size } \\
\cline { 2 - 4 } Condition & 2 & 3 & 4 \\
\hline 200-msec Interstimulus Interval & & & \\
Target old & 98.6 & 99.0 & 99.2 \\
Target new & 98.4 & 98.6 & 99.4 \\
0-msec Interstimulus Interval & & & \\
Target old & 96.3 & 97.6 & 98.1 \\
Target new & 98.8 & 99.7 & 99.4 \\
\hline
\end{tabular}

reliable effect of set size $[F(2,62)=4.16, p=.02]$. In addition, the participants were more accurate when the target was a new object (99.3\%) than when it was an old object $(97.3 \%)[F(1,31)=13.46, p<.001]$. This accuracy difference was consistent with the RT advantage when the target was a new object, reported below. Target type and set size did not interact $[F(2,62)=0.75, p=.48]$.

RT data are shown in Figure 5. In the 200-msec ISI condition, when the new object did not create a unique transient, no attention capture was observed. The search rate was not reliably different in the target-new $(22.3 \mathrm{msec} /$ item $)$ and target-old $(17.7 \mathrm{msec} /$ item $)$ conditions $(F<1)$. Collapsing across set size, responses were no faster in the target-new condition than in the target-old condition $(F<1)$.

In the 0 -msec ISI control condition, the new object created a unique onset transient, and the new object again captured attention. The search rate was shallower in the target-new condition $(8.3 \mathrm{msec} /$ item $)$ than in the targetold condition $(24.3 \mathrm{msec} /$ item $)[F(1,31)=10.7, p=$ .003]. Collapsing across set size, responses were faster in the target-new condition than in the target-old condition $[F(1,31)=217.1, p<.001]$.

Consistent with capture by the new object in the 0 -msec ISI condition, but not in the $200-\mathrm{msec}$ ISI condition, the slope data produced a reliable interaction between ISI condition and target type (old, new) $[F(1,31)=12.6, p<$ $.001]$.

The elimination of the pattern mask did not have any observable effect on attention capture across an ISI. Capture was observed only in the 0 -msec ISI condition, when the new object created a unique onset transient.

\section{EXPERIMENT 4}

In order to be considered a candidate for attention capture, a stimulus event must be detected efficiently across the visual field without significant capacity constraint (Yantis $\&$ Egeth, 1999). For a stimulus to draw attention away from a currently attended object or location (i.e., capture attention), detection of the stimulus cannot itself require focal attention. Efficient detection of this type generates pop-out in visual search, with detection largely independent of the set size of the search array (e.g., Treisman \& Gelade, 1980). A further condition for attention capture is that after detection, the stimulus must draw attention reflexively to the location where it appeared (Yantis \& Egeth, 1999). Note that even if a stimulus can be detected without focal attention, it need not capture attention: A single blue object embed- 
Table 4

Mean Accuracy (in Percentages) in Experiment 4

\begin{tabular}{ccccccc}
\hline & \multicolumn{6}{c}{ Set Size } \\
\cline { 2 - 7 } Condition & 2 & 3 & 4 & 6 & 10 & 16 \\
\hline 100-msec Interstimulus Interval & 97.3 & 96.2 & 96.9 & 95.9 & 92.4 & 86.9 \\
400-msec Interstimulus Interval & 98.6 & 97.3 & 97.4 & 95.1 & 95.2 & 86.4 \\
\hline
\end{tabular}

ded in a display of red objects can be detected efficiently and pops out in visual search, but there remains debate over whether such a color singleton involuntarily captures attention (Leber \& Egeth, 2006; Theeuwes, 2004).

The results of Experiments 1-3 suggest that a new object across an ISI does not draw attention reflexively. In Experiment 4, we tested whether a new object across an ISI meets even the more basic condition of efficient detection. Specifically, we tested whether a new object across an ISI pops out when the new object is explicitly the target of search. As in Experiments 1 and 2, a placeholder and search array were separated by a masked ISI. However, unlike in those earlier experiments, the new object after the ISI was the target on every trial. The participants simply had to detect the new object and identify it. They had every incentive to attend to the new object, and the conditions for observing pop-out search were therefore significantly less stringent than those in studies of attention capture. In Experiment 4, the new object did not have to capture attention to generate pop-out search; all that was required for pop-out search was that the new object could be detected efficiently. If a new object across an ISI can be detected efficiently and pops out, search slopes should be shallow ( $\sim-10 \mathrm{msec} / \mathrm{item}$; Wolfe,
2001). For comparison, when a new-object target created a unique onset in the 0-msec ISI conditions in Experiments 2 and 3, search slopes were 2.0 and $8.3 \mathrm{msec} /$ item, respectively. However, if the new object cannot be detected efficiently across an ISI, slopes should be consistent with a serial search operation (>25-35 msec/item; Wolfe, 2001). To provide a robust estimate of slope, we used a relatively large range of set sizes $(2,3,4,6,10$, and 16).

\section{Method}

Participants. Sixteen participants completed Experiment 4.

Stimuli and Apparatus. The background was gray, and the placeholders, letters, and fixation cross were black. Placeholders subtended $0.97^{\circ} \times 1.42^{\circ}$. In the preview display, block-letter- 8 placeholders were randomly assigned to locations within a $6 \times 6$ grid of squares, excluding the central four cells. Each cell subtended $2.27^{\circ} \times 2.27^{\circ}$. The preview display contained one fewer item than did the ultimate set size of $2,3,4,6,10$, or 16 items. In the search display, the target letter was either an "A" or an "F." Distractors were drawn randomly from the set "P," "E," "H," and "O." On each trial, a new letter was added to the search display at a randomly chosen position, and this item was always the target.

Procedure. The participants pressed a pacing button to initiate each trial, followed by a central fixation cross for $500 \mathrm{msec}$. The preview display was then presented for $1,000 \mathrm{msec}$, followed by

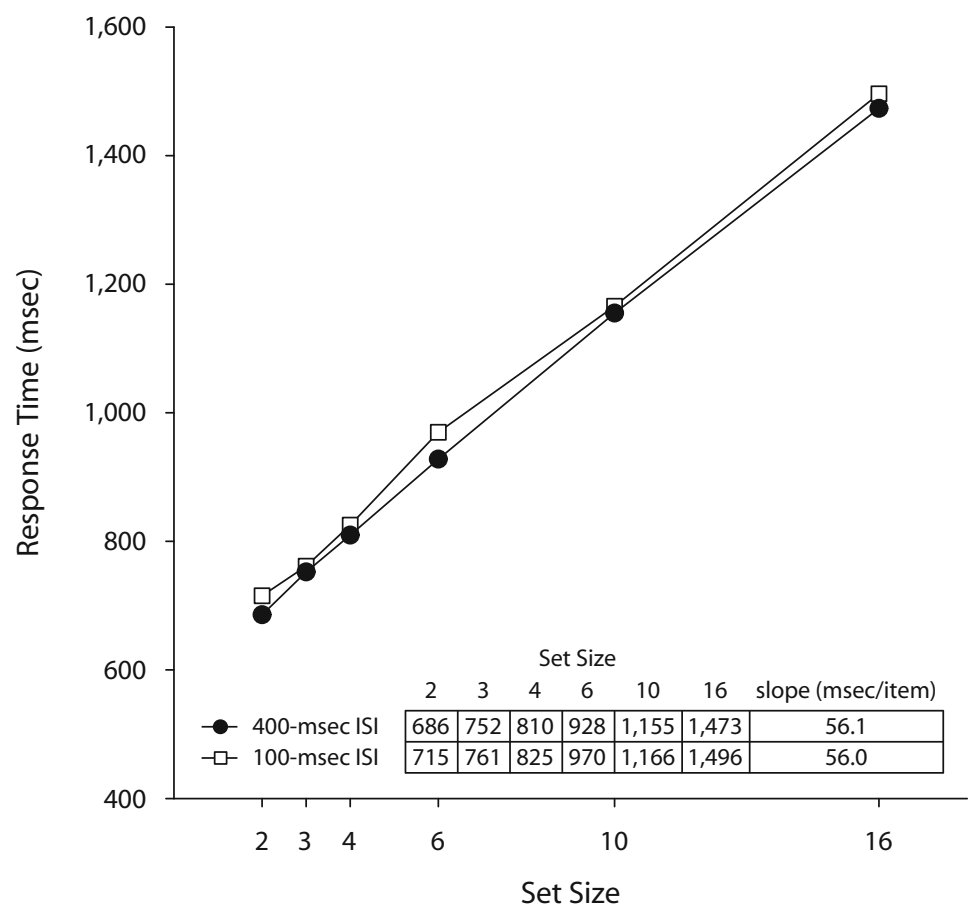

Figure 6. Experiment 4: Mean response time data as a function of interstimulus interval (ISI) duration and set size. 
a masked ISI of either 100 or $400 \mathrm{msec}$ (as used by Davoli et al., 2007), followed by the search display composed of letters. Using the index and middle fingers of the right hand, the participants pressed one of two buttons to indicate the presence of "A" or "F." Incorrect responses resulted in an error message and a short delay. The participants were instructed to respond as quickly and as accurately as possible. They were also instructed that, in the search array, there was always one new letter that appeared in a previously unoccupied location and that this letter was always the target, so their best strategy was to monitor for the new letter.

The experiment was a 2 (ISI) $\times 6$ (set size) $\times 2$ (target: "A" or "F") design. The participants first completed a practice session of 8 trials, drawn randomly from the full design, followed by 360 experiment trials divided evenly among the 24 conditions. Trial order was determined randomly.

\section{Results and Discussion}

The mean error rate was $5.4 \%$, with error rates increasing with increasing set size $[F(5,75)=11.3, p<.001$; see Table 4]. There was no effect of ISI $[F(1,15)=1.02, p=$ $.33]$ and no interaction between ISI and set size $(F<1)$.

Search times increased linearly with set size, and search was highly inefficient (see Figure 6). The mean search slope was $56.0 \mathrm{msec} / \mathrm{item}$, which was reliably greater than zero $[t(15)=7.97, p<.001]$. There was no reliable effect of ISI duration $[F(1,15)=2.99, p=.10]$ and no interaction between ISI duration and set size $(F<1)$. As is evident in Figure 6, the participants could not reliably locate the new object after the ISI, and they were forced to search serially through the array. ${ }^{5}$ Efficient detection, as indexed by pop-out search, is an entry condition that must be met for any stimulus attribute to be considered a candidate for attention capture (Yantis \& Egeth, 1999); in order to recruit attention, the detection of the stimulus attribute cannot itself require focal attention. Thus, a new object after an ISI cannot be considered to be a plausible candidate for a stimulus attribute that automatically captures attention.

\section{GENERAL DISCUSSION}

For a stimulus to capture attention, it must be detected efficiently across the visual field, and its presence must subsequently draw attention (Yantis \& Egeth, 1999). Many stimuli meet the first criterion. These include salient static features, such as unique color, shape, or orientation, and dynamic changes in luminance or motion. Some static feature differences additionally draw attention, depending on the stimulus properties and the nature of the task (Folk et al., 1992; Theeuwes, 1992). And several types of dynamic cues also draw attention efficiently to their location in a manner that is largely independent of task set (Abrams \& Christ, 2005; Enns et al., 2001; Franconeri \& Simons, 2003; Theeuwes, 1991; Thomas \& Luck, 2000; Yantis \& Jonides, 1984, 1990).

The present experiments tested the hypothesis that a particularly high-level dynamic event involuntarily captures attention: the appearance of a new object (Yantis, 2000; Yantis \& Hillstrom, 1994). Critically, according to the new-object hypothesis, it is the new object per se that attracts attention, independently of the luminance or motion change that typically accompanies the appearance of a new object in the visual field. According to one account (Yantis \& Hillstrom, 1994), the visual field is parsed initially, and a set of object files (Kahneman, Treisman, \& Gibbs, 1992) is established for visible objects. These representations must, at a minimum, allow for the representation of an object as a discrete entity persisting over time. Otherwise, there would be no basis upon which to distinguish new objects from old objects. If a new object is added to a scene, it is the one object without an existing object file representation, and the automatic process of creating a new object file triggers a shift of attention to that object. A transient signal is not required for this process to occur. As long as there is an object in the scene without an object file representation, one will be created, and the object will draw attention. In this view, it could be possible for a new object to capture attention across a blank ISI (or other perceptual disruption, such as a blink, eye movement, or brief occlusion), as long as the representations of previously visible objects are preserved across the ISI. ${ }^{6}$ After the ISI, the only object without an existing object file representation would be the new object, and that object would then capture attention.

As we have argued, the prediction of new-object capture across an ISI quickly runs into problems of capacity. The VWM system that maintains visual objects and locations across brief disruptions has a limited capacity (e.g., Luck $\&$ Vogel, 1997). If the number of objects in the visual field exceeds the capacity of VWM (which is true of most realworld environments), the locations and visual properties of all the old objects could not be remembered reliably across the ISI, and a new object added to the scene could not be detected efficiently. ${ }^{7}$ Failure to detect changes to scenes when detection depends on limited-capacity VWM has been observed consistently in the change blindness literature.

Moreover, problems of capacity arise for the new-object hypothesis even without the need to remember object information across a disruption such as an ISI. The newobject hypothesis depends on the ability to maintain object file representations for all currently visible objects. If there were any old objects in a scene that were not currently represented as object files, the absence of an object file representation would not serve to distinguish the new object from old objects, and the new object would not reliably draw attention. A real-world scene typically contains scores of individual objects, and it is well established that the capacity to maintain simultaneous, persisting object representations is limited (e.g., Pylyshyn \& Storm, 1988). As a result, it is unlikely that an object could be discriminated as new on the basis of object file representations, because the absence of an object file representation would be a property shared by many of the old objects in the scene.

Accounts of attention capture that depend on limitedcapacity systems of persisting object files (Yantis \& Hillstrom, 1994) or visual memory (Chua, 2009; Davoli et al., 2007) therefore do not provide a reliable means for detecting new objects in the types of complex environments characteristic of real-world vision. In contrast, unique sensory transients can be detected across the visual field regardless of the complexity of the scene, and they provide reliable 
cues to the appearance of new objects and to other salient environmental events (such as object motion or looming). Consistent with these observations, new objects introduced into complex scenes are not detected immediately and do not attract attention automatically when the transient associated with the new object is masked by saccadic suppression (Brockmole \& Henderson, 2005a, 2005b), and new objects do not capture attention in visual search paradigms when the transient created by the new object is masked by brief occlusion (Franconeri et al., 2005).

In the present study, we examined whether a new object captures attention during visual search if the new object is added to the search array across a blank ISI (Davoli et al., 2007). In this case, the new object does not create a unique onset transient, because all search items generate an onset transient after the ISI. In Experiment 1, we were unable to replicate the results of Davoli et al. suggestive of newobject capture across an ISI. In Experiments 2 and 3, new objects did not capture attention across an ISI, even when the paradigm was modified to be maximally sensitive to new-object capture across an ISI (by decreasing the ISI duration, by reducing the number of objects so as not to exceed VWM capacity, and by eliminating the potentially disruptive pattern mask used by Davoli et al., 2007). In contrast, we observed robust capture in Experiments 2 and 3 in the standard version of the attention capture paradigm (no ISI), in which the new object created a unique onset transient. Finally, in Experiment 4, we confirmed that the addition of a new object across an ISI is not detected reliably, even when the new object is always the target of search. If a new object across an ISI cannot be detected efficiently, it cannot automatically capture attention. Together, the present data highlight the importance of sensory transients in attention capture and support the claim that a unique transient is necessary for attention capture by a new object (Franconeri et al., 2005).

A second issue raised by the present study is that the instructions given to participants can influence the extent to which they strategically prioritize new objects. In Experiment $1 \mathrm{~B}$, we found that the instructions used by Davoli et al. (2007) did not necessarily cause participants to strategically monitor for new objects. However, directly encouraging the participants to attend to new objects in Experiment $1 \mathrm{C}$ produced shallower search slopes when the target was the new object. This manipulation mimicked a pattern of attentional capture, even though the effect was likely caused by voluntary orienting to new objects. Concerns about strategic prioritization have been amplified by another recent study of attention capture (Chua, 2009). Chua used the Franconeri et al. (2005) attention capture paradigm, in which a new object was introduced during a brief period of occlusion, masking the transient associated with its appearance. Again, the new object did not capture attention in the absence of a transient. In additional experiments, Chua found that when the new object was the target, slopes were consistent with efficient search if (1) participants knew precisely where the new object would appear on each trial and (2) participants were given strong incentive to strategically attend to the new object by always making it the target (similar to the present Experiment 4). Although Chua described these strategic effects as "attention capture," that description is imprecise. Capture refers to an involuntary event, and the term attention capture should be limited to circumstances in which attention is oriented involuntarily to a particular object or location. Chua's results are therefore entirely consistent with our evidence that capture by a new object requires a transient. Participants may be able to prioritize a new object strategically if they know exactly where it will appear and have strong incentive to attend to it, but new objects do not capture attention if the transient associated with the new object is masked by brief occlusion.

In general, for a paradigm to provide persuasive evidence about attention capture, it must meet three conditions, all of which were proposed originally by Yantis, Jonides, and colleagues (e.g., Yantis \& Jonides, 1984). We illustrate these conditions using examples from new-object capture, but they apply to any form of stimulus event that is hypothesized to capture attention involuntarily. First, the location of the new object should be unpredictable. If participants know where the new object will appear, they can strategically attend to the new-object location before search. If the new-object location is attended before the new object appears, it cannot be said to have drawn attention to itself involuntarily. Second, the new object cannot have a higher probability of being the search target than any of the other objects in the search array. If the new object has a higher probability of being the target, participants have an incentive to focus attention on that object voluntarily, so any observed prioritization cannot be attributed to involuntary capture. In fact, some of the strongest evidence for attention capture has come from paradigms in which the critical stimulus was never the target and capture was observed as a decrease in search efficiency when the critical stimulus was present (e.g., Remington, Johnston, \& Yantis, 1992; Theeuwes, 1992). Third, even if the new object is no more likely to be the target than are the other search elements, participants must not be able to construe any advantage to strategically attending to new objects.

Finally, we were unable to replicate the results of Davoli et al. (2007), despite implementing their experimental design and instructions in Experiment 1. The source of the difference is elusive. One possibility is that their result was simply a Type I error. Another possibility is that there yet remains an unidentified methodological difference between the two studies. Importantly, however, we were unable to find new-object capture across an ISI, despite generating increasingly more conducive conditions for the detection of new objects in Experiments 2-4, conditions significantly more liberal than those in the original Davoli et al. paradigm. In particular, new objects were not detected efficiently across an ISI even when the new object was the search target on every trial in Experiment 4. Thus, although we cannot pinpoint the source of the empirical difference between the two studies, we can be confident that the Davoli et al. results were unlikely to have been caused by involuntary attention capture by a new object across an ISI. 


\section{CONCLUSION}

The present data - in combination with those of Franconeri et al. (2005), Chua (2009), and Brockmole and Henderson (2005a, 2005b) - provide strong evidence that sensory transients play a central role in attention capture (see also Cole \& Kuhn, 2009) and that new objects do not capture attention without a unique sensory transient. If the transient typically generated by the appearance of a new object is masked by brief occlusion (Chua, 2009; Franconeri et al., 2005), an eye movement (Brockmole \& Henderson, 2005a, 2005b), or a brief ISI (the present study), the new object does not capture attention. This is not to say that transient events are the only events that capture attention. There remains debate about whether static singleton features, such as a unique color, recruit attention involuntarily (Leber \& Egeth, 2006; Theeuwes, 2004). However, in real-world cognition, visual transients are likely to play a central role in alerting the visual system to salient environmental events that lie outside the current, top-down focus of attention.

\section{AUTHOR NOTE}

Correspondence should be addressed to A. Hollingworth, Department of Psychology, University of Iowa, 11 Seashore Hall E, Iowa City, IA 52242-1407 (e-mail: andrew-hollingworth@uiowa.edu).

\section{REFERENCES}

Abrams, R. A., \& Christ, S. E. (2005). The onset of receding motion captures attention: Comment on Franconeri and Simons (2003). Perception \& Psychophysics, 67, 219-223.

Brockmole, J. R., \& Henderson, J. M. (2005a). Object appearance, disappearance, and attention prioritization in real-world scenes. Psychonomic Bulletin \& Review, 12, 1061-1067.

Brockmole, J. R., \& Henderson, J. M. (2005b). Prioritization of new objects in real-world scenes: Evidence from eye movements. Journal of Experimental Psychology: Human Perception \& Performance, 31, 857-868.

ChuA, F. K. (2009). A new object captures attention—but only when you know it's new. Attention, Perception, \& Psychophysics, 71, 699-711.

Cole, G. G., Kentridge, R. W., \& Heywood, C. A. (2004). Visual salience in the change detection paradigm: The special role of object onset. Journal of Experimental Psychology: Human Perception \& Performance, 30, 464-477.

Cole, G. G., \& Kunn, G. (2009). Appearance matters: Attentional orienting by new objects in the precueing paradigm. Visual Cognition, $17,755-776$.

Cole, G. G., Kunn, G., \& Liversedge, S. P. (2007). Onset of illusory figures attenuates change blindness. Psychonomic Bulletin \& Review, 14, 939-943.

Davoli, C. C., Suszko, J. W., \& Abrams, R. A. (2007). New objects can capture attention without a unique luminance transient. Psychonomic Bulletin \& Review, 14, 338-343.

DownING, P. E., \& DodDs, C. M. (2004). Competition in visual working memory for control of search. Visual Cognition, 11, 689-703.

Enns, J. T., Austen, E. L., Di Lollo, V., Rauschenberger, R., \& YANTIS, S. (2001). New objects dominate luminance transients in setting attentional priority. Journal of Experimental Psychology: Human Perception \& Performance, 27, 1287-1302.

Folk, C. L., Remington, R. W., \& Johnston, J. C. (1992). Involuntary covert orienting is contingent on attentional control settings. Journal of Experimental Psychology: Human Perception \& Performance, 18, 1030-1044.

Franconeri, S. L., Hollingworth, A., \& Simons, D. J. (2005). Do new objects capture attention? Psychological Science, 16, 275-281.
Franconeri, S. L., \& Simons, D. J. (2003). Moving and looming stimuli capture attention. Perception \& Psychophysics, 65, 999-1010.

Franconeri, S. L., Simons, D. J., \& Junge, J. A. (2004). Searching for stimulus-driven shifts of attention. Psychonomic Bulletin \& Review, 11, 876-881

HAYHOE, M. (2000). Vision using routines: A functional account of vision. Visual Cognition, 7, 43-64.

HollingwORTH, A. (2004). Constructing visual representations of natural scenes: The roles of short- and long-term visual memory. Journal of Experimental Psychology: Human Perception \& Performance, 30, 519-537.

HOLLingwORTH, A. (2005). The relationship between online visual representation of a scene and long-term scene memory. Journal of Experimental Psychology: Learning, Memory, \& Cognition, 31, 396-411.

HollingwORTH, A. (2008). Visual memory for natural scenes. In S. J. Luck \& A. Hollingworth (Eds.), Visual memory (pp. 123-162). New York: Oxford University Press.

Hollingworth, A. (2009). Two forms of scene memory guide visual search: Memory for scene context and memory for the binding of target object to scene location. Visual Cognition, 17, 273-291.

HollingWORTH, A., \& LUCK, S. J. (2009). The role of visual working memory (VWM) in the control of gaze during visual search. Attention, Perception, \& Psychophysics, 71, 936-949.

Hyun, J.-S., Woodman, G. F., Vogel, E. K., Hollingworth, A., \& LUCK, S. J. (2009). The comparison of visual working memory representations with perceptual inputs. Journal of Experimental Psychology: Human Perception \& Performance, 35, 1140-1160.

Jiang, Y., Olson, I. R., \& Chun, M. M. (2000). Organization of visual short-term memory. Journal of Experimental Psychology: Learning, Memory, \& Cognition, 26, 683-702.

Kahneman, D., Treisman, A., \& Gibbs, B. J. (1992). The reviewing of object files: Object-specific integration of information. Cognitive Psychology, 24, 175-219.

Land, M. F., Mennie, N., \& Rusted, J. (1999). Eye movements and the roles of vision in activities of daily living: Making a cup of tea. Perception, 28, 1311-1328.

Leber, A. B., \& Egeth, H. E. (2006). It's under control: Top-down search strategies can override attentional capture. Psychonomic Bulletin \& Review, 13, 132-138.

LuCK, S. J., \& VeCERA, S. P. (2002). Attention: From tasks to mechanisms. In S. Yantis (Ed.), Stevens' Handbook of experimental psychology: Vol. 1. Sensation and perception (pp. 235-286). New York: Wiley.

LUCK, S. J., \& VogeL, E. K. (1997). The capacity of visual working memory for features and conjunctions. Nature, 390, 279-281.

MitrofF, S. R., \& Alvarez, G. A. (2007). Space and time, not surface features, guide object persistence. Psychonomic Bulletin \& Review, 14, 1199-1204.

Olivers, C. N. L., Meijer, F., \& Theeuwes, J. (2006). Feature-based memory-driven attentional capture: Visual working memory content affects visual attention. Journal of Experimental Psychology: Human Perception \& Performance, 32, 1243-1265.

PASHLER, H. (1998). The psychology of attention. Cambridge, MA: MIT Press.

PhILliPs, W. A. (1974). On the distinction between sensory storage and short-term visual memory. Perception \& Psychophysics, 16, 283290.

Pylyshyn, Z. W., \& Storm, R. W. (1988). Tracking multiple independent targets: Evidence for a parallel tracking mechanism. Spatial Vision, 3, 179-197.

Remington, R. W., Johnston, J. C., \& Yantis, S. (1992). Involuntary attentional capture by abrupt onsets. Perception \& Psychophysics, $\mathbf{5 1}, 279-290$.

Schneider, W., Eschmann, A., \& Zuccolotto, A. (2002). E-Prime user's guide. Pittsburgh, PA: Psychology Software Tools.

Simons, D. J., \& Rensink, R. A. (2005). Change blindness: Past, present, and future. Trends in Cognitive Sciences, 9, 16-20.

Soto, D., Heinke, D., Humphreys, G. W., \& Blanco, M. J. (2005). Early, involuntary top-down guidance of attention from working memory. Journal of Experimental Psychology: Human Perception \& Performance, 31, 248-261.

Theeuwes, J. (1991). Exogenous and endogenous control of attention: 
The effect of visual onsets and offsets. Perception \& Psychophysics, 49, 83-90.

Theeuwes, J. (1992). Perceptual selectivity for color and form. Perception \& Psychophysics, 51, 599-606.

Theeuwes, J. (2004). Top-down search strategies cannot override attentional capture. Psychonomic Bulletin \& Review, 11, 65-70.

Thомas, S. J., \& LUCK, S. J. (2000). Multiple pathways to the automatic capture of attention. Unpublished manuscript, University of Iowa, Iowa City.

Torralba, A., Oliva, A., Castelhano, M. S., \& Henderson, J. M. (2006). Contextual guidance of eye movements and attention in realworld scenes: The role of global features in object search. Psychological Review, 113, 766-786.

Treisman, A. M., \& Gelade, G. (1980). A feature-integration theory of attention. Cognitive Psychology, 12, 97-136.

Wolfe, J. M. (2001). Asymmetries in visual search: An introduction. Perception \& Psychophysics, 63, 381-389.

Woodman, G. F., \& LuCK, S. J. (2007). Do the contents of visual working memory automatically influence attentional selection during visual search? Journal of Experimental Psychology: Human Perception \& Performance, 33, 363-377.

YANTIS, S. (2000). Goal directed and stimulus driven determinants of attentional control. In S. Monsell \& J. Driver (Eds.), Control of cognitive processes: Attention and performance XVIII (pp. 73-103). Cambridge, MA: MIT Press.

Yantis, S., \& EgEth, H. E. (1999). On the distinction between visual salience and stimulus-driven attentional capture. Journal of Experimental Psychology: Human Perception \& Performance, 25, 661-676.

Yantis, S., \& Gibson, B. S. (1994). Object continuity in apparent motion and attention. Canadian Journal of Experimental Psychology, 48, 182-204.

Yantis, S., \& Hillstrom, A. P. (1994). Stimulus-driven attentional capture: Evidence from equiluminant visual objects. Journal of Experimental Psychology: Human Perception \& Performance, 20, 95-107.

YANTIS, S., \& Jonides, J. (1984). Abrupt visual onsets and selective attention: Evidence from visual search. Journal of Experimental Psychology: Human Perception \& Performance, 10, 601-621.

YANTIS, S., \& Jonides, J. (1990). Abrupt visual onsets and selective attention: Voluntary versus automatic allocation. Journal of Experimental Psychology: Human Perception \& Performance, 16, 121-134.

\section{NOTES}

1. We use the term $V W M$ to describe both short-term memory for objects and short-term memory for locations. Under optimal conditions, VWM appears to be limited to approximately four objects (Luck \& Vogel, 1997) and six locations (Jiang, Olson, \& Chun, 2000).

2. Note that we are claiming not that attention capture will occur across an ISI if the number of objects is within VWM capacity but, rather, that the visual system does not have sufficient resources to detect a new object if the number of objects exceeds VWM capacity.

3. We thank Chris Davoli for providing additional details of the Davoli et al. (2007) method and instructions so that we could ensure that Experiment 1 matched the Davoli et al. experiments as closely as possible.

4. The Yantis and Gibson (1994) results are consistent with an account in terms of new-object capture, but they are equally consistent with an account in terms of capture by the onset transient generated by the reappearing object (Franconeri et al., 2005), since the transient created by the disappearance and reappearance of the object would have been less salient at very short ISIs.

5. It is informative to compare the search rate when the target is always the new object to the search rate when the target is always an old object. In a follow-up experiment $(N=12)$, we added a block to the Experiment 4 design in which all objects (including the target) were old after the ISI and, thus, participants were forced to search serially through the array. Search was inefficient in this target-old condition $(61.3 \mathrm{msec} /$ item) and in the replication of the Experiment 4 target-new condition ( $48.6 \mathrm{msec} / \mathrm{item})$. The relatively small slope difference between the two conditions was likely to have been caused by the fact that in the targetnew conditions, participants could remember a handful of old-object locations across the ISI and deprioritize these in the subsequent search (since they would never contain the target).

6 . Note that Yantis and colleagues never made claims about attention capture across an ISI per se. Our point applies to the Davoli et al. (2007) extension of the new-object framework.

7. With a longer time to view a scene, one can certainly encode information from a much larger number of objects (Hollingworth, 2004, 2005), but this long-term memory representation is unlikely to support online attention capture (Brockmole \& Henderson, 2005b).

(Manuscript received July 24, 2009;

revision accepted for publication February 13, 2010.) 